\title{
Acute Lymphoblastic Leukemia Manifesting as Acute Vogt-Koyanagi-Harada Disease
}

\author{
Hee Kyung Yang, Hyeong Gon $\mathrm{Yu}, \mathrm{MD}, \mathrm{PhD}$ \\ Department of Ophthalmology, Seoul National University College of Medicine, Seoul Artificial Eye Center, \\ Seoul National University Hospital Clinical Research Institute, Seoul, Korea
}

\begin{abstract}
We describe a case of bilateral exudative retinal detachment associated with prodromal symptoms simulating the presentation of acute Vogt-Koyanagi-Harada disease that was eventually diagnosed as acute lymphoblastic leukemia. A 42-year-old man presented with sudden visual loss in both eyes for two weeks. He complained of intermittent headache, neck stiffness and tinnitus for a month. His best-corrected visual acuities were 20/200 in both eyes. Fluorescein angiography, optical coherence topography and indocyanine green angiography featured bilateral serous retinal detachments. A clinical diagnosis of incomplete type Vogt-Koyanagi-Harada disease was considered. However, complete blood cell count showed a marked increase in the number of white blood cells and bone marrow examination revealed precursor B cell lymphoblastic leukemia. The patient started on induction chemotherapy. A week later, his best-corrected visual acuities were $20 / 25$ and the serous retinal detachments were nearly absorbed in both eyes. Bilateral exudative retinal detachment associated with neurologic and auditory abnormalities may be a presenting sign of acute lymphoblastic leukemia. Clinicians should be aware of the possibility of leukemia in such patients. Korean J Ophthalmol 2009;23:325-328 @ 2009 by the Korean Ophthalmological Society.
\end{abstract}

Key Words: Exudative retinal detachment, Leukemia, Vogt-Koyanagi-Harada disease

Exudative retinal detachment, a very rare ocular finding in leukemia, has only been described in a few cases and has not been previously reported in South Korea. ${ }^{1-8}$ This is the first case of acute lymphoblastic leukemia presenting with bilateral exudative retinal detachment associated with prodromal symptoms of neurologic and auditory abnormalities simulating the presentation of acute stage Vogt-Koyanagi-Harada (VKH) disease. This case is also the first to document indocyanine green angiography (ICGA) findings of serous retinal detachments in acute lymphoblastic leukemia.

\section{Case Report}

A previously healthy 42-year-old man presented with sudden visual loss in both eyes for two weeks. The decline in left eye visual acuity had begun two weeks before, followed by the right eye one week later. He was initially diagnosed with central serous chorioretinopathy (CSC) at another clinic. His visual acuities worsened, however, and he came to our clinic for a second opinion. His medical history was obvious for interm-

Received: August 8, 2008 Accepted: November 11, 2009

Reprint requests to Hyeong Gon Yu, MD, PhD.The Department of Ophthalmology, Seoul National University Hospital and Medical Research Center, Seoul National University, Seoul, Korea. Tel: 82-22072-2437, Fax: 82-2-741-3187, E-mail: hgonyu@snu.ac.kr ittent headache, neck stiffness and tinnitus for a month. Otherwise, he had no history of any systemic disease, ocular trauma or surgery. His best-corrected visual acuities were 20/200 in both eyes, and there was no chamber reaction in either eye. Vitreous cells were positive at grade $2+$ in both eyes and fundus examination showed bilateral serious retinal detachments with Elschnig's spots in the left eye (Fig. 1). Optical coherence tomography (OCT) revealed macular subretinal serous fluids in both eyes, with a minimum foveal thickness of $944 \mathrm{um}$ in the right eye and $818 \mathrm{um}$ in the left eye (Fig. 2). Fluorescence angiography (FA) in the early phase showed delayed choroidal filling and hypofluorescence in the macula, followed by mid-phase punctuate hyperfluorescence at the level of the retinal pigment epithelium clustered along the boundaries of the detached retina (Fig. 3A). Late phase FA was apparent for diffuse late leakage and fluorescence staining in the area of exudative retinal detachment (Fig. 3B). ICGA in the early phase featured broad hypofluorescence due to a choroidal filling defect in the posterior pole (Fig. 3C), which was followed by mid-phase fluorescence blockage in the area of exudative retinal detachment (Fig. 3D). A clinical diagnosis of incomplete type VKH was considered. However, systemic evaluation revealed increased white blood cell counts $(290,000 / \mu \mathrm{L})$, with an abnormally increased percentage of blast cells (89\%). Bone marrow examination confirmed the diagnosis of precursor $\mathrm{B}$ cell lymphoblastic leukemia. He received induction chemotherapy of vincristine, prednisolone, daunorubicin, 1-aspa- 

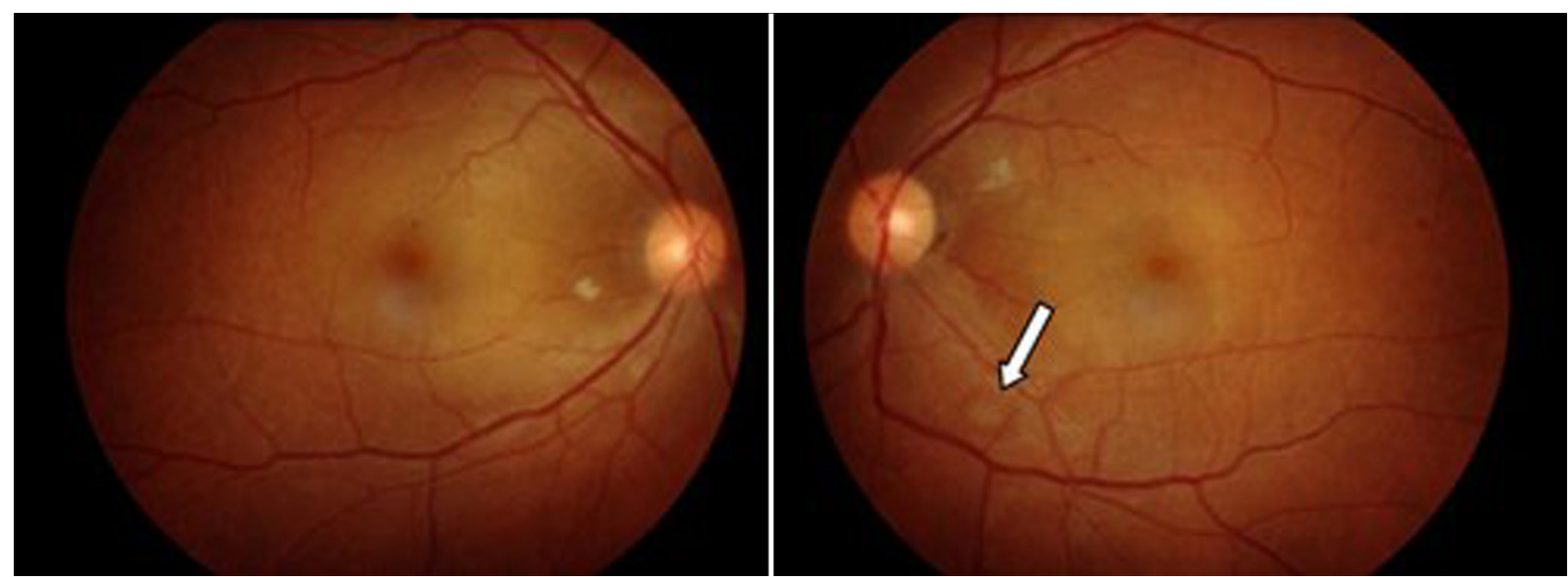

Fig. 1. Initial presentation. Fundus photo of both eyes showing exudative retinal detachment. An Elschnig's spot (white arrow) is seen in the left eye.

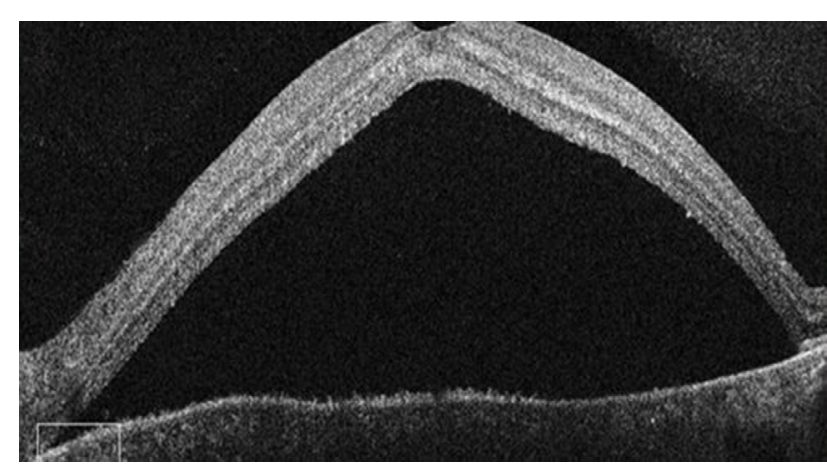

Fig. 2. Optical coherence tomography of the macula in both eyes showed massive subretinal exudative fluid in the posterior pole. No subretinal septa were observed.

raginase (VPDL) and high-dose steroid therapy (prednisolone $60 \mathrm{mg}$ daily).

A week later, his best corrected visual acuities were 20/25, and there was no chamber reaction in both eyes. Vitreous cells were reduced to grade $1+$ in both eyes and the serous retinal detachments were nearly absorbed in both eyes. Newly developed Roth spots and cotton wool patches near the optic disc were found in both eyes. FA no longer showed leakages of stippled hyperfluorescence (Fig. 4). Although his bestcorrected visual acuities improved after chemotherapy, the patient died of pulmonary hemorrhage and multiorgan failure during immunosuppression, 18 days after starting chemotherapy.

\section{Discussion}

Visual loss after choriocapillary occlusion in leukemia was first reported by Zimmerman in $1964 .{ }^{1}$ Beyond that, exudative retinal detachment in leukemia has been reported in only a few cases world-wide, as a presenting sign of the disease or the first sign of relapse. ${ }^{1-8}$ This CSC-like serous retinal detachment in leukemia has not been previously reported in South Korea. This is also the first case showing characteristic prodromal symptoms of neurologic and auditory abnormalities. The presence of such symptoms preceding bilateral exudative retinal detachment is a typical feature of VKH disease, fulfilling the diagnostic criteria of incomplete VKH disease as long as there is no underlying systemic disease. ${ }^{9}$

The mechanism of serous retinal detachment in leukemia has been thought to be choroidal ischemia and secondary retinal pigment epithelial dysfunction. ${ }^{3,4,6}$ The choroid is the most frequently involved ocular tissue in leukemia. Leukemic cell infiltration or hematologic disturbances may cause partial occlusion of the choriocapillaries and delay of choroidal circulation. ${ }^{10}$ Secondary dysfunction of the Bruch's membrane and retinal pigment epithelium may ultimately develop into serous retinal detachments of the macula. ${ }^{3,8,11,12}$ Ocular histologic findings by autopsy in leukemic patients have documented the features of choroidal thickening and leukemic cell infiltration that lead to choroidal ischemia. ${ }^{10}$ Although we did not have histologic evidence, the fact that systemic chemotherapy induced a rapid remission of exudative retinal detachment, consistent with previous reports, suggests leukemic cell infiltration as the underlying pathology. ${ }^{3,4,7}$

This case is the first to document early and late ICGA findings of serous retinal detachments in acute lymphoblastic leukemia. The early phase of ICGA demonstrated choroidal ischemia, especially in the posterior pole, whereas choroidal filling in the normal eye always begins in the macula with initial fluorescing of subfoveal choroidal arterioles. ${ }^{13}$ After the midphase, choroidal fluorescence was blocked by serous retinal detachments and the details of retinal and choroidal vessels were obscured. Fluorescence blockage by serous retinal fluid is also seen in CSC or VKH disease, usually in the late phase of ICGA. ${ }^{14}$ However, the typical hypofluorescent dark dots in the mid-phase usually observed in CSC or VKH disease were not seen in our case. ${ }^{14}$ This inconsistency suggests a different pathophysiology, and the penetration of ICG dye may have been reduced by highly concentrated fluid in the subretinal 


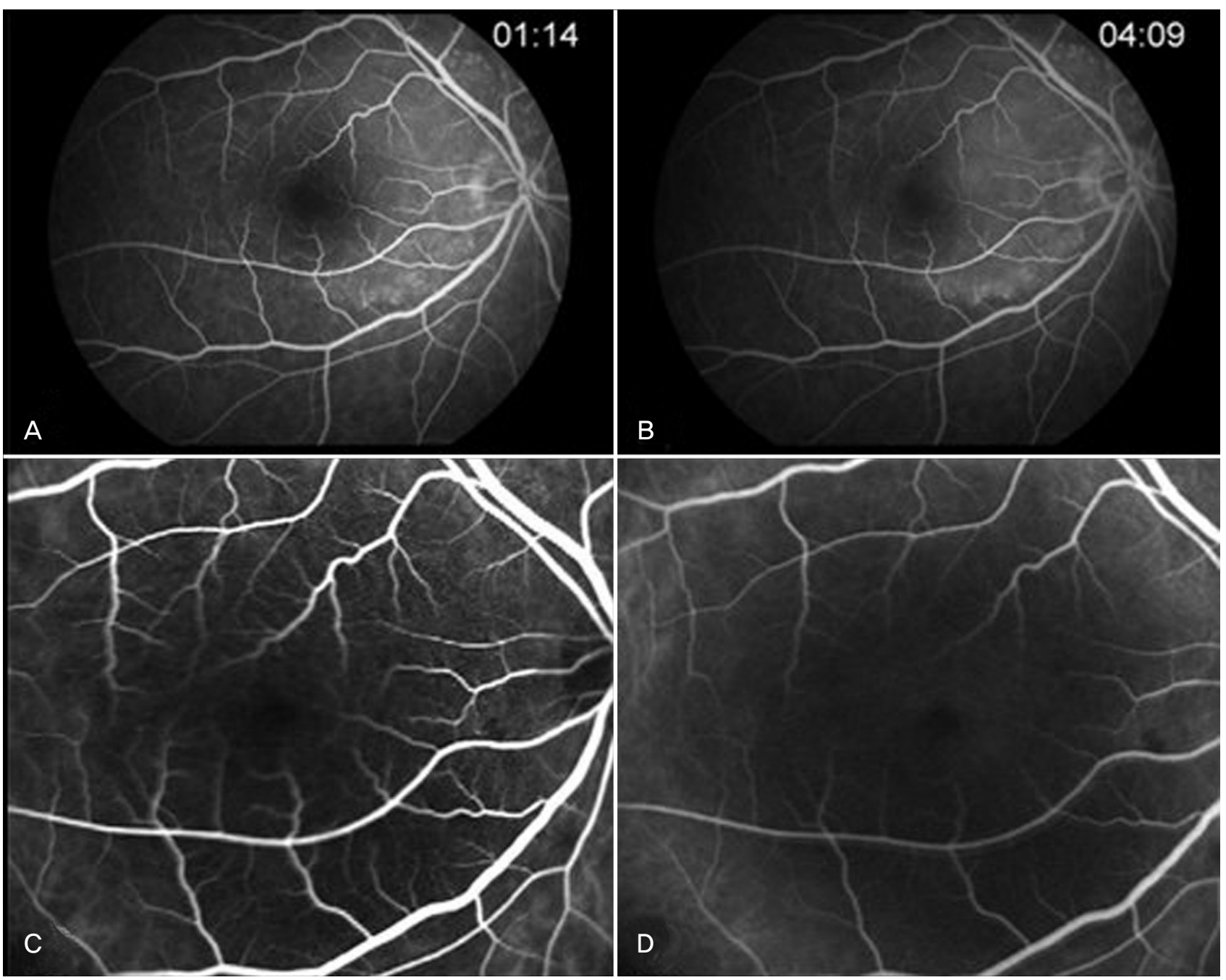

Fig. 3. Fluorescence angiography (FA) and indocyanine green angiography (ICGA) of the right eye. (A) Mid-phase FA showed patchy choroidal filling and multiple punctate hyperfluorescent lesions. (B) Late-phase FA showed diffuse leakage and unilobular fluorescence pooling. (C) Early-phase ICGA $(35 \mathrm{sec})$ showed a hypofluorescent choroidal filling defect in the macula. (D) Mid-phase ICGA (4 min) showed a larger area of macular hypofluorescence due to fluorescence blockage in the areas of exudative retinal detachment.
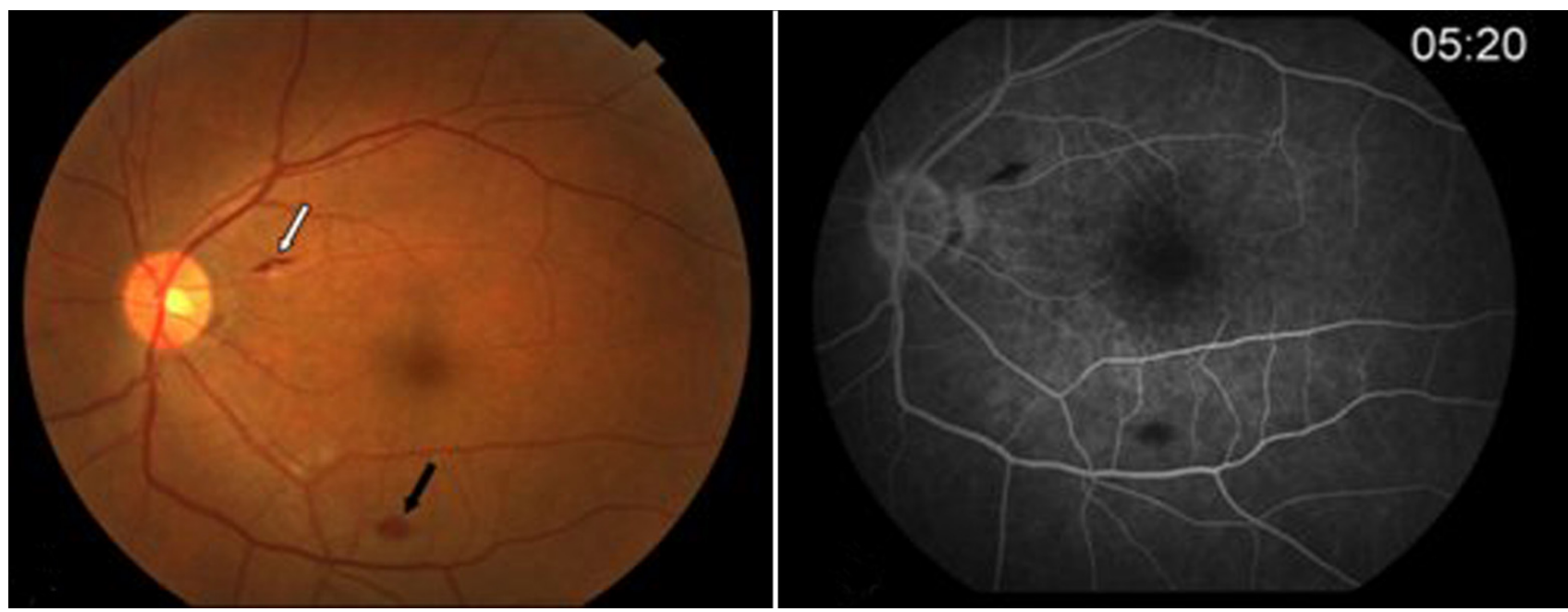

Fig. 4. One week after chemotherapy. (A) Fundus photo of the left eye showed nearly absorbed serous retinal detachments with newly appeared retinal hemorrhage (black arrow) and Roth spots (white arrow). (B) Fluorescence angiography of the left eye no longer showed multilple hyperfluorescent lesions in the late phase. 
space along with thick cellular infiltrates in the choroid.

Prodromal symptoms of headache, neck stiffness and tinnitus, such as in our case, followed by bilateral serous retinal detachment, may misdirect to the clinical diagnosis of incomplete type VKH disease. ${ }^{9}$ However, several features are helpful in making the differential diagnosis. First, anterior uveitis was absent in our case, which is frequently seen in VKH disease. ${ }^{15}$ Second, FA findings of mid-phase multiple hyperfluorescent dots were clustered along the boundaries of the detached retina, sparing the macula; in VKH disease such dots are usually distributed in the posterior pole. ${ }^{14}$ Third, late-phase dye pooling in FA showed a unilobular CSC-like feature, different from the multi-lobulated pooling seen in VKH disease. ${ }^{16}$ Fourth, OCT did not reveal any of the subretinal septa generally observed in VKH disease. ${ }^{16}$ Finally, typical ICGA findings of VKH disease were absent in our case, such as mid-phase multiple hypofluorescent dots in the posterior pole. ${ }^{14}$ The prodromal symptoms that misdirected the diagnosis may have been caused by leukemic infiltration into the cerebrospinal fluid and microvasculopathy due to hyperleukocytosis. ${ }^{17,18}$

Leukemia may present when there is sudden visual loss due to exudative retinal detachment, and the ophthalmologist may be the first clinician to encounter such patients. A thorough systemic evaluation should be performed in patients presenting with bilateral exudative retinal detachment, especially in those who report certain systemic symptoms.

\section{References}

1. Zimmerman LE, Thoreson HT. Sudden loss of vision in acute leukemia: a clinicopathologic report of two unusual cases. Surv Ophthalmol 1964;146:467-73.

2. Stewart MW, Gitter KA, Cohen G. Acute leukemia presenting as a unilateral exudative retinal detachment. Retina 1989;9:110-4.

3. Chen MT, Wu HJ. Acute leukemia presenting as diabetes insipidus and bilateral exudative retinal detachment: a case report. Kaohsiung J Med Sci 2001;17:150-5.

4. Fackler TK, Bearelly S, Odom T, et al. Acute lymphoblastic leukemia presenting as bilateral serous macular detachments. Retina 2006;26:710-2.

5. Hine JE, Kingham JD. Myelogenous leukemia and bilateral exudative retinal detachment. Ann Ophthalmol 1979;11:186772.

6. Kincaid MC, Green WR, Kelley JS. Acute ocular leukemia. Am J Ophthalmol 1979;87:698-702.

7. Malik R, Shah A, Greaney MJ, Dick AD. Bilateral serous macular detachment as a presenting feature of acute lymphoblastic leukemia. Eur J Ophthalmol 2005;15:284-6.

8. Paydas S, Soylu MB, Disel U, et al. Serous retinal detachment in a case with chronic lymphocytic leukemia: no response to systemic and local treatment. Leuk Res 2003;27:557-9.

9. Read RW, Holland GN, Rao NA, et al. Revised diagnostic criteria for Vogt-Koyanagi-Harada disease: report of an international committee on nomenclature. Am J Ophthalmol 2001;131:64752 .

10. Leonardy NJ, Rupani M, Dent G, Klintworth GK. Analysis of 135 autopsy eyes for ocular involvement in leukemia. Am J Ophthalmol 1990;109:436-44.

11. Gaudric A, Sterkers M, Coscas G. Retinal detachment after choroidal ischemia. Am J Ophthalmol 1987;104:364-72.

12. Spaide RF, Goldbaum M, Wong DW, et al. Serous detachment of the retina. Retina 2003;23:820-46.

13. Ito YN, Mori K, Young-Duvall J, Yoneya S. Aging changes of the choroidal dye filling pattern in indocyanine green angiography of normal subjects. Retina 2001;21:237-42.

14. Oshima Y, Harino S, Hara Y, Tano Y. Indocyanine green angiographic findings in Vogt-Koyanagi-Harada disease. Am J Ophthalmol 1996;122:58-66.

15. Fang W, Yang P. Vogt-koyanagi-harada syndrome. Curr Eye Res 2008;33:517-23.

16. Yamaguchi Y, Otani T, Kishi S. Tomographic features of serous retinal detachment with multilobf seroye pooling in aeroyeVogtKoyanagi-Harada disease. Am J Ophthalmol 2007;144: 260-5.

17. Demopoulos A, DeAngelis LM. Neurologic complications of leukemia. Curr Opin Neurol 2002;15:691-9.

18. Resende LS, Coradazzi AL, Rocha-Junior C, et al. Sudden bilateral deafness from hyperleukocytosis in chronic myeloid leukemia. Acta Haematol 2000;104:46-9. 